\title{
Talin is required to increase stiffness of focal molecular complex in its early formation process
}

$\operatorname{AUTHOR}(\mathrm{S})$ :

Nakao, Nobuhiko; Maki, Koichiro; Mofrad, Mohammad K. R.; Adachi, Taiji

\section{CITATION:}

Nakao, Nobuhiko ...[et al]. Talin is required to increase stiffness of focal molecular complex in its early formation process. Biochemical and Biophysical Research Communications 2019, 518(3): 579-583

ISSUE DATE:

2019-10-20

URL:

http://hdl.handle.net/2433/261016

\section{RIGHT:}

(c) 2019. This manuscript version is made available under the CC-BY-NC-ND 4.0 license

http://creativecommons.org/licenses/by-nc-nd/4.0/:; The full-text file will be made open to the public on 20 October 2020 in accordance with publisher's 'Terms and Conditions for Self-Archiving.; This is not the published version. Please cite only the published version.この論文は出版社版でありません。引用の際には出版社版をご確認ご利用ください。 
Talin is required to increase stiffness of focal molecular complex in its early formation process.

Nobuhiko Nakao ${ }^{\mathrm{a}, \mathrm{b}}$, Koichiro Maki ${ }^{\mathrm{c}, \mathrm{d}}$, Mohammad R. K. Mofrad ${ }^{\mathrm{e}, \mathrm{f,g}}$ and Taiji Adachi ${ }^{\mathrm{a}, \mathrm{b}, \mathrm{h}}$

${ }^{\text {a }}$ Department of Micro Engineering, Graduate School of Engineering, Kyoto University, 53 Shogoin Kawahara-cho, Sakyo, Kyoto 606-8507, Japan

${ }^{\mathrm{b}}$ Institute for Frontier Life and Mechanical Sciences, Kyoto University, 53 Shogoin Kawahara-cho, Sakyo, Kyoto 606-8507, Japan

${ }^{c}$ Helsinki Institute of Life Science, University of Helsinki, Yliopistonkatu 3, Helsinki, FI00290, Finland

${ }^{\mathrm{d}}$ Department of Mechanical Engineering, Graduate School of Engineering, The University of Tokyo, 7-3-1 Hongo, Bunkyo, Tokyo 113-8656, Japan

${ }^{\mathrm{e}}$ Department of Bioengineering, University of California, Berkeley, CA94720, USA

${ }_{\mathrm{f}}^{\mathrm{f}}$ Department of Mechanical Engineering, University of California, Berkeley, CA94720-1762, USA

${ }^{\mathrm{g}}$ Molecular Biophysics and Integrative Bioimaging Division, Lawrence Berkeley National Lab, CA94720, Berkeley, USA

${ }^{\mathrm{h}}$ Department of Mammalian Regulatory Network, Graduate School of Biostudies, Kyoto University, 53 Shogoin Kawahara-cho, Sakyo, Kyoto 606-8507, Japan

\section{Corresponding author}

Taiji Adachi, Ph.D., Professor

Email address: adachi@infront.kyoto-u.ac.jp 


\section{Abstract}

For cellular adaptation in mechanical environments, it is important to consider transmission of forces

from the outside to the inside of cells via a focal molecular complex. The focal molecular complex,

which consists of integrin, talin, vinculin and actin, is known to form in response to a force applied via

the extra-cellular matrix (ECM). In the early formation process of the complex, the complex-actin

connection is reinforced. These structural changes of the nascent complex result in an increase in its

mechanical integrity and overall stiffness, possibly leading to the maturation of the nascent complex

by enhancing force transmission. In this study, we hypothesized that the complex component talin is a

crucial factor in increasing the stiffness of the nascent complex. To test the hypothesis, we used atomic

force microscopy (AFM) to measure the stiffness of the nascent complex using a probe coated with

fibronectin. Stiffness measurements were conducted for intact and talin knocked-down cells. Our

results demonstrated that talin was required to increase the stiffness of the nascent complex, which

could be caused by the reinforced connection between the complex and actin filaments mediated by

talin.

\section{Key words:}

Focal molecular complex, Talin, Stiffness, Atomic force microscopy, Mechanotransduction 


\section{Introduction}

Cell adaptation to mechanical environments is triggered by force transmission from the outside to the inside of the cells [1]. Force is transmitted via a focal molecular complex that connects the extracellular matrix (ECM) and the cytoskeleton [2]. The focal molecular complex, consisting of integrin, talin, vinculin, and actin [3], is formed when the cell is subjected to a force via the ECM [4]. With regard to the formation of the nascent complex, early molecular events are particularly important because the complex is formed based on its initial structure. A previous study suggested that the nascent complex matures by reinforcing the complex-actin connection, and this complex disappear without such a reinforcement [5]. Therefore, the reinforced connection is thought to play a key role in the transmission of force generated outside of cells.

During force transmission, the stiffness of the complex, which is a measure of its mechanical integrity, is regarded as a pivotal factor. During its initial formation stage, a structural change in the complex can certainly lead to a change in its stiffness. Thus, an increase in the stiffness of the nascent complex enhances the force transmitted from the ECM to the cytoskeleton, possibly leading to maturation of the nascent complex through the opening of mechanosensitive ion channels [6] and/or the activation of signaling molecules via deformation of the cytoskeleton [7].

In a cell adhesion region, component proteins form a nascent complex by binding with each other within $50 \mathrm{~s}$ under an external force transmitted via the ECM $[5,8,9]$. For complex formation, the ECMintegrin binding first causes conformational changes in the integrin molecule [10], and the outside-in 
signaling initiates complex formation [11]. Then, talin and actin localize into the cell adhesion region to form an integrin-talin-actin complex [12]. Eventually, to bind to vinculin, talin changes its conformation by exposing its cryptic vinculin-binding site [13-16]. These sequential molecular events lead to a reinforced connection between integrin and the actin filament $[16,17]$. Since talin works as an adapter protein to connect integrin with actin filament, this connection is closely related to a change in the stiffness of the complex. In this study, we hypothesized that talin contributes to increasing the stiffness of the nascent complex. To test the hypothesis, we investigated the role of talin on the stiffness of the nascent complex using atomic force microscopy (AFM).

\section{Materials and Methods}

2.1. Cell culture

In this study, we used mouse pre-osteoblast MC3T3-E1 cells (Riken Cell Bank), which retains the cell surface receptor integrin $\beta 1$ [18]. Cells were cultured in a minimum essential medium $\alpha$ (MEM $\alpha$; Gibco) containing 10\% fetal bovine serum (FBS; Gibco) and 1\% antibiotic-antimycotic (Nacalai tesque) in a humidified incubator at $37^{\circ} \mathrm{C}$ under a $5 \% \mathrm{CO}_{2}$ atmosphere.

\subsection{Modification of AFM probe}

OMCL-TR400PSA (spring constant $=0.02 \mathrm{~N} / \mathrm{m}$, tip radius $=15 \mathrm{~nm}$, Olympus) was used as the

AFM probe. To coat the probe with fibronectin (i.e., form an FN probe), organic matters on the probe 
tip were cleaned using a vacuum plasma processing system (STREX). Then, for silane coupling, the tip was put in 2\% 3-aminopropyl-triethoxysilane (APTES; Sigma-Aldrich)/pure water for $15 \mathrm{~min}$, and then rinsed with pure water. The tip was immersed in $1 \mathrm{mM}$ linker protein maleimidepolyethyleneglycol-N-hydroxyl-succinimide (Maleimide-PEG-NHS; NANOCS)/pure water for 30 min, and then rinsed. Finally, the tip surface was modified in $100 \mathrm{nM}$ fibronectin (EMD Millipore)/PBS for 60 min, and then rinsed. Afterwards, to quench the remaining Maleimide residues, the tip was placed in 2-mercaptoethanol (Wako) for 60 min. For a negative control group, an AFM probe coated with BSA (BSA probe) was fabricated by modifying 4\% BSA after Maleimide-PEGNHS.

\subsection{Immunofluorescence imaging of integrin on AFM probe}

To visualize the integrin attached on the tip of the FN probe after it was in contact with the cells, the tip surface was fixed with paraformaldehyde and blocked with 3\% BSA/PBS. Subsequently, the tip surface was coated with antibodies for integrin staining and then rinsed with PBS. The rat anti-mouse integrin $\beta 1$ antibody (Abcam) and Alexa Fluor 488-conjugated donkey anti-rat IgG antibody (Abcam) were used as the primary and secondary antibodies, respectively. Total internal reflection fluorescence microscopy (TIRFM; Olympus) was employed to detect fluorescence of the antibody, and the results were finally analyzed using the image processing software ImageJ (NIH). 


\subsection{Talin 1 knock down}

To inhibit expression of talin in cells, we added plasmids (pSilencer 3.0-H1) to knock down talin 1 and plasmids (pCAGGS-EGFP) to express the green fluorescent protein (GFP) by using a transfection reagent FuGENE HD (Promega) dissolved in Opti-MEM (Gibco). The GFP positive cells were used as talin 1 knocked-down cells in the experiments. The culture medium was then replaced by a transfecting mixture solution. After $2 \mathrm{~h}$ of incubation in the transfecting mixture solution at $37^{\circ} \mathrm{C}$ in a $5 \% \mathrm{CO}_{2}$ atmosphere, the cells were cultured in a fresh normal culture medium for $46 \mathrm{~h}$ at $37^{\circ} \mathrm{C}$ with $5 \% \mathrm{CO}_{2}$. The culture time was set such that the highest transfection efficiency could be obtained as described in a previous study [19].

\subsection{AFM stiffness measurement}

Stiffness measurement for the focal molecular complex using AFM (NanoWizard 3, JPK) was performed on cells cultured for more than $2 \mathrm{~h}$. The AFM probe approached the cell surface at $1 \mu \mathrm{m} / \mathrm{s}$ by extending a piezo actuator with a constant indentation force $(F=-100 \mathrm{pN})$ using a feedback control system (Fig. 1 A, black). Then, the probe and the cell surface were kept in contact for different contact time $T[\mathrm{~s}](0 \mathrm{~s}, 5 \mathrm{~s}, 10 \mathrm{~s}, 15 \mathrm{~s}, 20 \mathrm{~s}, 25 \mathrm{~s}$, and $30 \mathrm{~s})$ (Fig. 1 B). After a contact time $T$ had passed, the probe was pulled away from the cell surface at $1 \mu \mathrm{m} / \mathrm{s}$ by retracting the piezo actuator (Fig. $1 \mathrm{~A}$, purple). Accordingly, a force against piezo height curve (i.e. force curve) was obtained. For each contact time $T$, force curves were obtained from 8-10 cells. A 25-point grid was drawn on the cell 
within an area of $10 \mu \mathrm{m} \times 10 \mu \mathrm{m}$, and measurements were taken at each point on the grid.

To determine the stiffness of the complex, the force curves were analyzed using JPKSPM Data Processing (JPK). The probe tip height was determined from the piezo height, force and its spring constant. The tilt of the curves caused by thermal drift was corrected by subtracting the slope of the curves after rupture. Then, the force after rupture was set to $F=0$ as the origin. The origin of extension $\Delta L[\mathrm{~nm}]$ was defined as the tip height when the force reached $0 \mathrm{pN}$. After these processes, the force curves (as shown in Fig. 1 C) were analyzed. A positive curve, in which the focal molecular complex was successfully formed, was defined using a threshold of a maximum force $F_{\max }[\mathrm{pN}]$. The threshold was determined as $\mu+2 \sigma(\mu$ : mean, $\sigma$ : S.D. $)(67.5 \mathrm{pN})$ at $0 \mathrm{~s}$ contact. The maximum force $F_{\max }$ was obtained from the curve within $500 \mathrm{~nm}$ of $\Delta L$, which is several times as long as the length of the complex $(\sim 100 \mathrm{~nm})$ [3]. The stiffness of the focal molecular complex was defined as stiffness $K$ $[\mathrm{pN} / \mathrm{nm}]$ that is the slope of curves $(0 \mathrm{~nm} \leq \Delta L \leq 100 \mathrm{~nm})$ (Fig. $1 \mathrm{D})$. For these calculations, the numerical computational software Scilab (INRIA) was used.

\section{Results}

\subsection{Formation of fibronectin-integrin binding}

We examined whether fibronectin-integrin binding occurred when the FN probe came into contact with the cells. To confirm the presence of integrin residue on the tip of the post-contact FN probe, TIRFM imaging was conducted. Fluorescence images in Figs. 2 A and B represented fluorescence 
spots on the tip of non- and post-contact FN probes, respectively. Fluorescence intensity on the tip of the post-contact FN probe was higher than that on the non-contact FN probe (Fig. 2 A, B lower), indicating that integrin was detected on the tip of the post-contact probe. This implies that fibronectinintegrin binding occurred when the FN probe came into contact with the cells.

\subsection{Formation of integrin-talin-actin filament complex}

We verified the formation of integrin-talin-actin filament complex inside the cell after the FN probe came into contact with the cells. To evaluate the complex formation, positive curves, in which the complex was successfully formed, were obtained based on maximum force $F_{\max }$ following the two procedures discussed: First, a threshold of maximum force $F_{\max }$ was defined as $\mu+2 \sigma$ ( $\mu$ : mean, $\sigma$ : S.D.) at $0 \mathrm{~s}$ contact (Fig. $3 \mathrm{~A}$ ). As contact solely for $0 \mathrm{~s}$ is extremely short, the fibronectin-integrin binding did not occur, and hence, the complex could not be formed. The positive curve was then defined as a curve in which the maximum force $F_{\max }$ exceeded the threshold. The complex formation was evaluated as a time-dependent variation in proportion of positive curves to total force curves.

For intact cells, the proportion of positive curves was linearly increased as fitted by the black line ( $p=0.0033$, Fig. 3 B), which we confirmed that the complex was successively induced by interaction with fibronectin. We then compared the proportion of intact and talin knocked-down cells to verify the formation of the talin-containing molecular complex on the intact cells. The proportion for talin knocked-down cells (Fig. 3 B, gray bar) was evidently lesser than that for intact cells. This result 
indicates that talin was present in the molecular complex formed inside the intact cells. Moreover, we tested whether the complex formation was triggered by fibronectin-integrin binding. For the negative control group, the proportion for BSA probe (Fig. 3 B, white bar) was evidently lesser than that for FN probe, indicating that fibronectin-integrin binding was required for the complex formation. Taken together, these results imply that the integrin-talin-actin filament complex was formed after the probecell contact.

\subsection{Increase in stiffness $K$ requiring talin}

To investigate the effect of talin on the stiffness of nascent focal molecular complex, stiffness $K$ (Fig.1 D) was measured for each contact time $T$ on the intact cells and talin knocked-down cells (Fig. 4). Regarding the intact cells, stiffness $K$ for 10-to-30-s contact significantly increased compared with that for $5 \mathrm{~s}$ contact (Two-way ANOVA test followed by Tukey-Kramer test, $p<0.001$ ). Stiffness $K$ for talin knocked-down cells, in contrast, was less than that for the intact cells (Two-way ANOVA test, $p<$ 0.001). These data demonstrated that talin was required to increase the stiffness of nascent focal molecular complex.

\section{Discussion}

Our novel method using AFM stiffness measurements first revealed that talin was required to increase the stiffness of the complex in its early process. The maturation of focal molecular complex 
has a major influence on cellular adaptation to the mechanical environment [20-22]. A previous research suggested that the maturation of the nascent complex depends on the reinforced complexactin filament connection [4]. This reinforcement is thought to enhance a force transmission function of the complex to open stretch-activated ion channels [5] and to send biochemical signals via the deformation of cytoskeleton [6], leading to the maturation of the complex. Although the stiffness of the complex plays a central role on the force transmission, previous studies have not focused on the stiffness of the nascent complex. This study first reported the involvement of talin in the increase in the stiffness of the complex during its early formation process.

A previous study suggested that talin led to the reinforced connection between nascent focal molecular complex and actin filament via its conformational changes. The molecular dynamics simulation demonstrated that a talin rod undergoes conformational changes to expose hidden vinculin binding sites under external tensile force [14]. In addition, an in vitro molecular experiment suggested that the talin-actin complex binds to vinculin by applying tensile force on the complex [15]. Moreover, a cellular experiment indicated that vinculin was colocalized with talin in a stretched cell [16]. These studies suggested that the connection between focal molecular complex and actin filament could be reinforced, by connecting more actin filaments to talin via vinculin $[23,24]$. In our experiment, talin, vinculin and actin began to localize in the adhesion area within $10 \mathrm{~s}$ which is consistent with the observations made in previous studies [4,9]. Taken together, the focal nascent complex-actin filament connection in our experiment was probably reinforced after conformational changes of talin molecule. 
To directly reveal the structural changes of the complex involving talin, vinculin and actin, their behavior should be visualized when the AFM probe comes into contact with the cells. By recruiting fluorescence microscopy and fluorescence probe in the AFM system, the localization of these proteins, conformational changes of the talin molecule and talin-vinculin binding can be visualized during the contact [25]. This helps verify the relation between the behavior of the proteins and stiffness of the complex. Thus, when exploring further studies, the introduction of a fluorescence system to our current AFM system will reveal the dynamics of the components underlying our results. Furthermore, the coupling of AFM to a fluorescence system will investigate modulation of force transmission by increase in the stiffness of the nascent complex and effects of the subsequent biochemical signaling on the complex maturation.

The AFM stiffness measurement for the nascent molecular complex is expected to help determine how its components affect various cell activities. For instance, the nascent focal molecular complex during cell migration generates more traction force than a matured complex [26]. During the development of the growth cone of a neuron, the cadherin-based nascent complex also generates the traction force [27]. As another cell activity, osteocyte senses the external force via the integrin-based molecular complex on narrow cell processes [28]. In cell behaviors represented above, the stiffness of the focal complex is thought to contribute to the force transmission for the cell traction or mechanotransduction. Moreover, the force transmission varies depending on the component proteins of the complexes (e.g. integrin $\beta 1$ or $\beta 3$ ) because each protein exhibits a different binding strength 
[29,30]. Employing AFM stiffness measurement helps better understand the cell activities regarding the force transmission function generated by different complexes components.

\section{References}

[1] P.P. Provenzano, P.J. Keely, Mechanical signaling through the cytoskeleton regulates cell proliferation by coordinated focal adhesion and Rho GTPase signaling, J. Cell Sceince. 124 (2011) 1195-1205. doi:10.1242/jcs.067009.

[2] K.A. Demali, X. Sun, G.A. Bui, Force Transmission at Cell-Cell and Cell-Matrix Adhesions, Biochemistry. 53 (2014) 7706-7717. doi:10.1021/bi501181p.

[3] J. Liu, Y. Wang, W.I. Goh, H. Goh, M.A. Baird, S. Ruehland, S. Teo, N. Bate, D.R. Critchley, M.W. Davidson, Talin determines the nanoscale architecture of focal adhesions, Proc. Natl.

Acad. Sci. U. S. A. 112 (2015) E4864-E4873. doi:10.1073/pnas.1512025112.

[4] D. Riveline, E. Zamir, N.Q. Balaban, U.S. Schwarz, T. Ishizaki, S. Narumiya, Z. Kam, B.

Geiger, A.D. Bershadsky, Focal Contacts as Mechanosensors : Externally Applied Local

Mechanical Force Induces Growth of Focal Contacts by an mDia1-dependent and, J. Cell Biol. 153 (2001) 1175-1185.

[5] C.K. Choi, M. Vicente-Manzanares, J. Zareno, W. A., Leanna, A. Mogilner, A.R. Horwitz, Actin and $\alpha$-actinin orchestrate the assembly and maturation of nascent adhesions in a myosin NIH Public Access, Nat. Cell Biol. 10(9) (2008) 1039-1050. doi:10.1038/ncb1763. 
[6] K. Hayakawa, H. Tatsumi, M. Sokabe, Actin stress fibers transmit and focus force to activate mechanosensitive channels, J. Cell Sci. 121(4) (2008) 496-503. doi:10.1242/jcs.022053.

[7] B. Han, X. Bai, M. Lodyga, J. Xu, B.B. Yang, S. Keshavjee, M. Post, M. Liu, Conversion of Mechanical Force into Biochemical Signaling, J. Cell Biol. 279 (2004) 54793-54801. doi:10.1074/jbc.M406880200.

[8] L. Tan, T. Meyer, B. Pfau, T. Hofmann, T.W. Tan, D. Jones, Rapid vinculin exchange dynamics at focal adhesions in primary osteoblasts following shear flow stimulation, $\mathrm{J}$. Musculoskelet. Neuronal Interact. 10 (2010) 92-99.

[9] C.G. Galbraith, K.M. Yamada, M.P. Sheetz, The relationship between force and focal complex development, J. Cell Biol. 159 (2002) 695-705. doi:10.1083/jcb.200204153.

[10] F.G. Giancotti, E. Ruoslahti, Integrin Signaling, Sceince. 285 (1999) 1028-1033.

[11] M. Kim, C. V. Christopher, T.A. Springer, Bidirectional Transmembrane Signaling by Cytoplasmic Domain Separation in Integrins, Science (80-. ). 301 (2003) 1720-1725.

[12] G.Y. Jiang, G. Giannone, D.R. Critchley, E. Fukumoto, M.P. Sheetz, Two-piconewton slip bond between fibronectin and the cytoskeleton depends on talin, Nature. 424 (2003) 334-337. doi:10.1038/nature01794.1.

[13] K. Maki, N. Nakao, T. Adachi, Nano-mechanical characterization of tension-sensitive helix bundles in talin rod, Biochem. Biophys. Res. Commun. 484 (2017) 372-377. doi:10.1016/j.bbrc.2017.01.127. 
[14] S.E. Lee, R.D. Kamm, M.R.K. Mofrad, Force-induced activation of Talin and its possible role in focal adhesion mechanotransduction, J. Biomech. 40 (2007) 2096-2106. doi:10.1016/j.jbiomech.2007.04.006.

[15] C. Ciobanasu, B. Faivre, C. Le Clainche, Actomyosin-dependent formation of the mechanosensitive talin-vinculin complex reinforces actin anchoring, Nat. Commun. 5 (2014) 3095. doi:10.1038/ncomms4095.

[16] H. Hirata, H. Tatsumi, C.T. Lim, M. Sokabe, Force-dependent vinculin binding to talin in live cells : a crucial step in anchoring the actin cytoskeleton to focal adhesions, Am. J. Physiol. Physiol. 306 (2014) C607-620. doi:10.1152/ajpcell.00122.2013.

[17] J.D. Humphries, P. Wang, C. Streuli, B. Geiger, M.J. Humphries, C. Ballestrem, Vinculin controls focal adhesion formation by direct interactions with talin and actin, J. Cell Biol. 179 (2007) 1043-1057. doi:10.1083/jcb.200703036.

[18] F.M. Pavalko, N.X. Chen, C.H. Turner, D.B. Burr, S. Atkinson, Y. Hsieh, J. Qiu, R.L. Duncan, Fluid shear-induced mechanical signaling in MC3T3-E1 osteoblasts requires cytoskeletonintegrin interactions, Am. J. Physiol. Physiol. 275 (1998) C1591-C1601.

[19] S. Yamano, J. Dai, A.M. Moursi, Comparison of Transfection Efficiency of Nonviral Gene Transfer Reagents, Mol. Biotechnol. 46 (2010) 287-300. doi:10.1007/s12033-010-9302-5.

[20] C.S. Chen, J. Tan, J. Tien, MECHANOTRANSDUCTION AT CELL-MATRIX AND CELLCELL CONTACTS, Annu. Rev. Biomed. Eng. 6 (2004) 275-302. 
doi:10.1146/annurev.bioeng.6.040803.140040.

[21] S. Saleem, J. Li, S. Yee, G.F. Fellows, C.G. Goodyer, R. Wang, $\beta 1$ integrin/FAK/ERK signalling pathway is essential for human fetal islet cell differentiation and survival, J. Pathol. 219 (2009) 182-192. doi:10.1002/path.

[22] S.M. Ponik, F.M. Pavalko, Formation of focal adhesions on fibronectin promotes fluid shear stress induction of COX-2 and PGE2 release in MC3T3-E1 osteoblasts, J. Appl. Physiol. 97 (2004) 135-142.

[23] A.R. Menkel, M. Kroemker, P. Bubeck, M. Ronsiek, G. Nikolai, B.M. Jockusch, Characterization of an F-actin-binding Domain in the Cytoskeletal Protein Vinculin, J. Cell Biol. 126 (1994) 1231-1240.

[24] J. Golji, M.R.K. Mofrad, The Interaction of Vinculin with Actin, PLOS Comput. Biol. 9 (2013) e1002995. doi:10.1371/journal.pcbi.1002995. doi: 10.1371/journal.pcbi.1002995.

[25] K. Maki, S. Han, Y. Hirano, S. Yonemura, T. Hakoshima, T. Adachi, Real-time TIRF observation of vinculin recruitment to stretched $\alpha$-catenin by AFM, Sci. Rep. 8 (2018) 1575. doi:10.1038/s41598-018-20115-8.

[26] K.A. Beningo, M. Dembo, I. Kaverina, J.V. Small, Y. Wang, Nascent Focal Adhesions Are Responsible for the Generation of Strong Propulsive Forces in Migrating Fibroblasts, J. Cell Biol. 153 (2001) 881-887.

[27] L. Bard, C. Boscher, M. Lambert, R.-M. Mege, D. Choquet, O. Thoumine, A Molecular Clutch 
between the Actin Flow and N-Cadherin Adhesions Drives Growth Cone Migration, J.

Neurosci. 28 (2008) 5879-5890. doi:10.1523/JNEUROSCI.5331-07.2008.

[28] P. Cabahug-zuckerman, R.F.S. Jr, R.J. Majeska, M.M. Thi, D.C. Spray, S. Weinbaum, M.B.

Schaffler, Potential Role for a Specialized b3 Integrin-Based Structure On Osteocyte Processes

in Bone Mechanosensation, J. Orthop. Res. 36 (2017) 642-652. doi:10.1002/jor.23792.

[29] P. Roca-cusachs, N.C. Gauthier, A. Del Rio, M.P. Sheetz, Clustering of $\alpha 5 \beta 1$ integrins

determines adhesion strength whereas $\alpha v \beta 3$ and talin enable mechanotransduction, Proc. Natl.

Acad. Sci. U. S. A. 106 (2009) 16245-16250.

[30] T. Adachi, Y. Aonuma, M. Tanaka, M. Hojo, T. Takano-yamamoto, H. Kamioka, Calcium response in single osteocytes to locally applied mechanical stimulus : Differences in cell process and cell body, J. Biomech. 42 (2009) 1989-1995. doi:10.1016/j.jbiomech.2009.04.034.

\section{Acknowledgement}

This work was supported by Advanced Research and Development Programs for Medical Innovation

(AMED-CREST), by elucidated of mechanobiological mechanisms and their application to the development of innovative medical instruments and technologies from Japan Agency for Medical Research and Development (AMED). We are grateful to Kazunori Nakajima and Kanehiro Hayashi for generously providing us with plasmids for talin 1 knock-down experiment. We would also like to thank Jeonghyun Kim for proofreading the manuscript. 


\section{Figure/Table Legends}

Fig. 1 AFM stiffness measurement. (A) Schematic of AFM stiffness measurement. The AFM probe approached the cell surface by extending a piezo actuator. After the probe-cell contact, the probe was pulled away by retracting the actuator. (B) The contact state was retained using constant force $(F=$ $100 \mathrm{pN}$ ) for contact time $T$ [s]. (C) Typical force curve. The intersection point represents the origin of the curve. The origin of force $F$ was determined as force after rupture. The origin of extension $\Delta L$ was defined as the tip height when force reached $0 \mathrm{pN}$. (D) Maximum force $F_{\max }[\mathrm{pN}]$ was measured within $500 \mathrm{~nm}$ of $\Delta L$. Stiffness $K[\mathrm{pN} / \mathrm{nm}]$ was calculated by linear approximation of the curve $(0 \mathrm{~nm}$ $\leq \Delta L \leq 100 \mathrm{~nm})$.

Fig. 2 Integrin $\beta 1$-fibronectin binding. Fluorescence images of (A) non- and (B) post-contact (contact time $T=30 \mathrm{~s}$ ) FN probe. The fluorescence intensity in the both images was profiled along with the red lines. The yellow lines indicate the outline of cantilevers. The blue and red dashed lines indicate the position of the probe.

Fig. 3 Formation of integrin-talin-actin filament complex for contact time T. (A) Time-dependent variations in maximum force $F_{\max }[\mathrm{pN}]$ for intact cells. Black points indicate the mean. Error bars indicate S.D.. A positive curve was defined as the force curve, in which maximum force $F_{\max }$ excesses $\mu+2 \sigma(\mu=$ mean, $\sigma=$ S.D. $)$ at 0 s contact. (B) Proportion of positive curve to total curves was shown 
with respect to each contact time $T$ for intact cells, talin 1 knocked-down cells and BSA probe. Linear regression analysis indicated that the proportion for intact cells significantly increased with contact time $T(p<0.01)$.

Fig. 4 Increase in stiffness $K$ requiring talin. Stiffness $K$ for intact and talin 1 knocked-down cells was plotted with respect to each contact time $T$. On the right side of the plots, mean and S.D. are shown. ANOVA test indicated that talin 1 knock-down had a significant effect on stiffness $K(p<0.001)$. Tukey-Kramer test indicated that stiffness $K$ for 10-to-30 s contact was significantly larger than that for $5 \mathrm{~s}$ contact in AFM stiffness measurements on intact cells $(* p<0.001)$. 
A

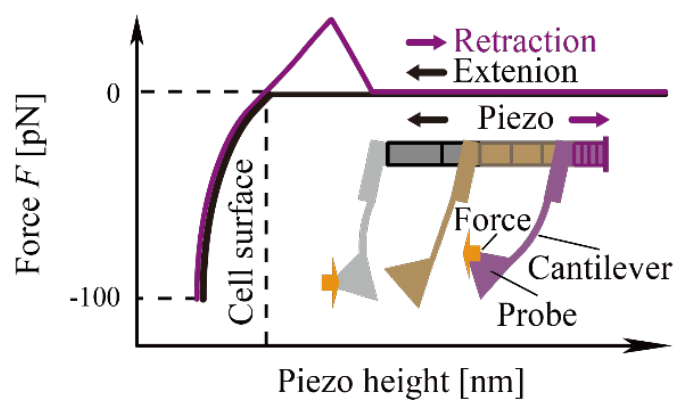

C

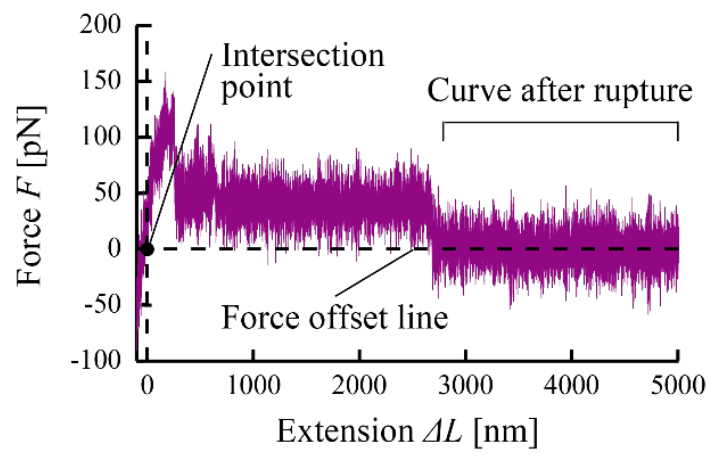

B

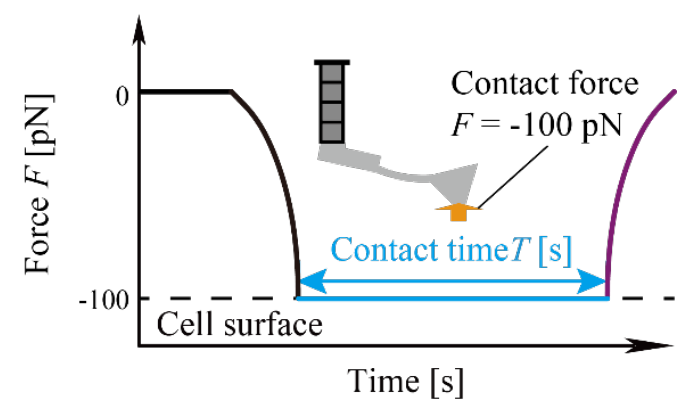

D

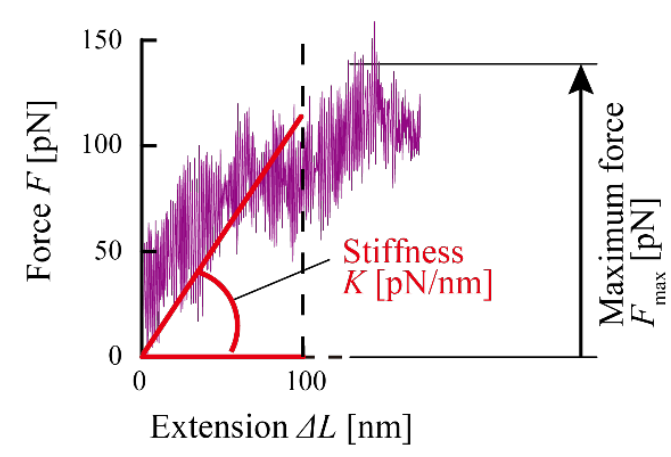

Fig. 1 
A

B

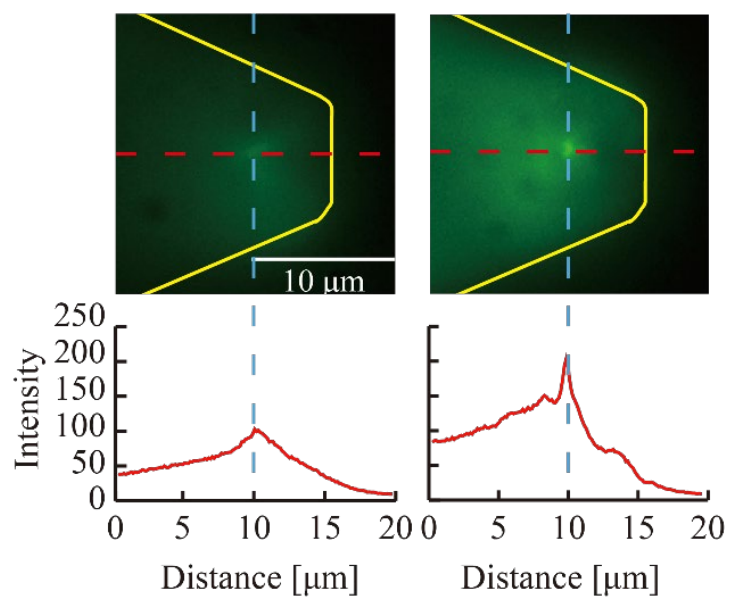

Fig. 2 
A

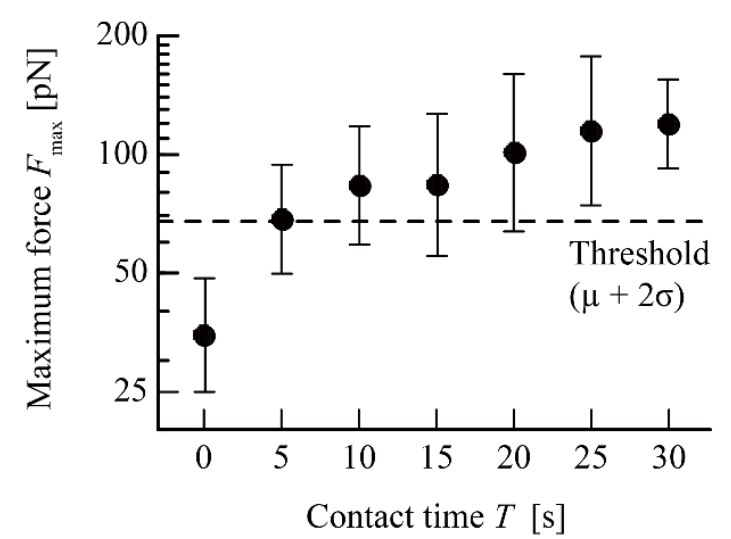

B

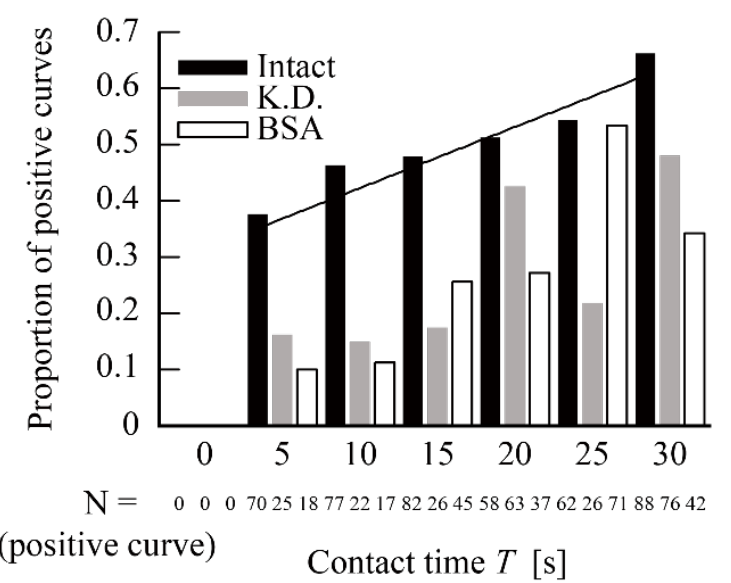

Fig. 3 


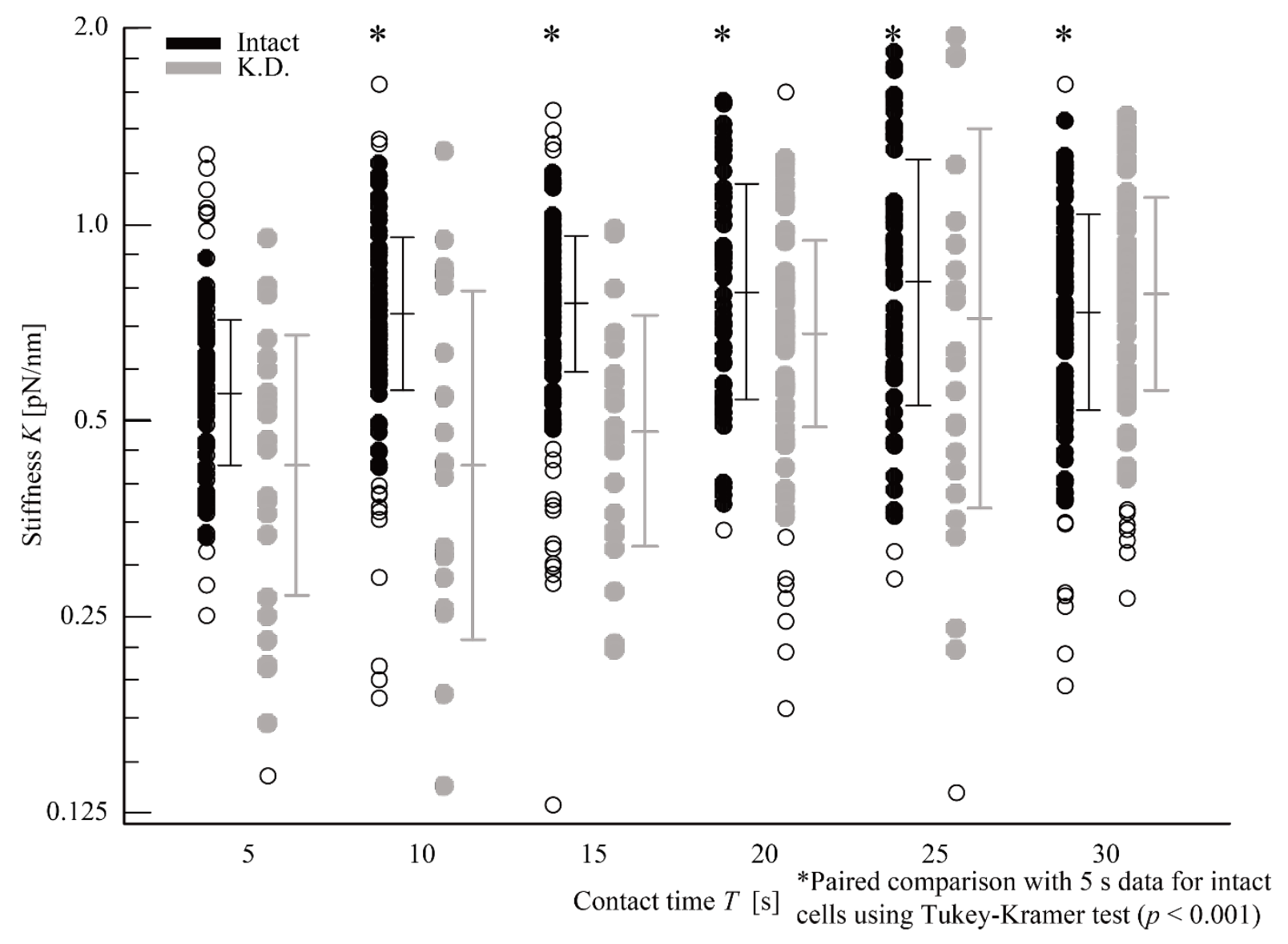

Fig. 4 